British Journal of Nutrition (2021), 126, 923-932

doi:10.1017/S0007114520004808

(C) The Author(s), 2020. Published by Cambridge University Press on behalf of The Nutrition Society. This is an Open Access article, distributed under the terms of the Creative Commons Attribution licence (http://creativecommons.org/licenses/by/4.0/), which permits unrestricted re-use, distribution, and reproduction in any medium, provided the original work is properly cited.

\title{
Dietary quality is associated with reduced risk of diabetes among adults in Northern China: a cross-sectional study
}

Siyue $\operatorname{Tan}^{1} \dagger$, Haiwen $\mathrm{Lu}^{2} \dagger$, Ruier Song ${ }^{1}$, Jing Wu³ $\mathrm{Wu}^{3}$ Mingming Xue ${ }^{4}$, Yonggang Qian ${ }^{5}$, Wenrui Wang ${ }^{5 *}$ and Xuemei Wang ${ }^{1 *}$

${ }^{1}$ Department of Health Statistics, School of Public Health, Inner Mongolia Medical University, Hohbot 010110, Inner Mongolia Autonomous Region, People's Republic of China

${ }^{2}$ Department of Medical Imaging, the Affiliated Hospital of Inner Mongolia Medical University, Inner Mongolia Medical University, Hohbot 010110, Inner Mongolia Autonomous Region, People's Republic of China

${ }^{3}$ Department of Chronic and Non-Communicable Disease Control and Prevention, National Center for Chronic and NonCommunicable Disease Control and Prevention, Chinese Center for Disease Control and Prevention, Beijing 100050, People's Republic of China

${ }^{4}$ Department of Physiology, School of Basic Medicine, Inner Mongolia Medical University, Hohbot 010110, Inner Mongolia Autonomous Region, People's Republic of China

${ }_{5}^{5}$ Department of Chronic Disease Control and Prevention, Inner Mongolia Center for Disease Control and Prevention, Hohhot 010031, Inner Mongolia Autonomous Region, People's Republic of China

(Submitted 8 July 2020 - Final revision received 31 October 2020 - Accepted 19 November 2020 - First published online 1 December 2020)

\section{Abstract}

In this study, we analysed the prevalence of diabetes in Inner Mongolia and explored the relationship between dietary patterns and diabetes using the Chinese Dietary Balance Index-16 (DBI-16). This study was a surveillance survey of Chronic Disease and Nutrition Monitoring among Chinese Adults in Inner Mongolia in 2015. Dietary data were collected using the 24-h dietary recall and weighing method over three consecutive days. Dietary quality was evaluated via the DBI-16. A generalised linear model was used to examine the associations between the DBI-16 and dietary patterns. The relationship between dietary patterns and diabetes was analysed using logistic regression. In Inner Mongolia, the diabetes prevalence was $8.5 \%$ and the estimated standardised prevalence was $6.0 \%$. Four major dietary patterns were identified: 'meat/dairy products', 'traditional northern', 'high cereal/tuber' and 'high-salt/alcohol'. Generalised linear models showed that the 'meat/dairy product' pattern was relatively balanced $\left(\beta_{\mathrm{LBS}}=-1.993, \beta_{\mathrm{HBS}}=-0.206, \beta_{\mathrm{DQD}}=-2 \cdot 199\right.$; all $\left.P<0.05\right)$ and was associated with a lower diabetes risk (OR $0.565 ; 95 \% \mathrm{CI}$ $0.338,0.945 ; P<0.05)$ after adjusting for potential confounders. The other three dietary patterns (i.e. 'traditional northern', 'high cereal/tuber' and 'high-salt/alcohol') exhibited relatively unbalanced dietary quality and were unassociated with diabetes risk. Diabetes prevalence in Inner Mongolia was moderate. The dietary quality of the 'meat/dairy product' pattern was relatively balanced and was correlated with a decreased risk of diabetes prevalence, suggesting that dietary quality may help decrease diabetes prevalence and provide a suggestion for local dietary guidelines.

Key words: Diabetes: Dietary patterns: Dietary quality: Chinese Dietary Balance Index-16

Diabetes is a serious public health problem. A study published in The Lancet showed that the number of people with diabetes worldwide increased from 108 million to 422 million in 1980$2014^{(1)}$. According to the 2019 International Diabetes Federation Western Pacific report, approximately $116 \cdot 4$ million adults aged 20-79 years in China have diabetes, representing a regional prevalence of $10 \cdot 9 \%^{(2)}$. A cross-sectional survey showed an $8.9 \%$ prevalence of diabetes in China, which is higher than that of South Korea $(5.7 \%)$ and Japan $(4.8 \%)^{(3)}$. Additionally, patients with diabetes are prone to developing chronic illnesses such as IHD, stroke, chronic kidney disease, peripheral artery disease, specific cancers and tuberculosis ${ }^{(4)}$. Preventing or delaying the onset of diabetes is a top priority for global public health.

Abbreviations: DBI-16, Dietary Balance Index-16; DQD, diet quality distance; HBS, higher-bound score; LBS, lower-bound score.

* Corresponding authors: Xuemei Wang, fax +471 6653892, email wangxm_zsu@163.com; Wenrui Wang, email wr821@163.com

$\dagger$ These authors contributed equally to this work 
Dietary factors are closely related to the development of diabetes. Among them, dietary intake is a major modifier in diabetes management ${ }^{(5)}$. Increased consumption of a healthy diet reduces the risk of diabetes ${ }^{(6)}$; therefore, the association between dietary patterns and diseases is gaining attention. Prospective observational studies and clinical trials have evidenced the importance of a healthy diet in preventing and managing diabetes $^{(7)}$. Examples include the Mediterranean-style diet, which emphasises synergy among various nutrients and foods ${ }^{(8)}$, and the Dietary Approaches to Stop Hypertension diet, which plays an important role in glycaemic control in long-term interventions ${ }^{(9)}$. One study found that adherence to the American Dietary Guidelines may reduce the risk of major chronic diseases $^{(10)}$. Scholars often use the Alternate Healthy Eating Index to evaluate the dietary habits of local populations ${ }^{(11,12)}$. The Alternate Healthy Eating Index enables assessing the relationship between diet and chronic diseases. Additionally, a meta-analysis revealed that the Mediterranean diet, Dietary Approaches to Stop Hypertension and Alternate Healthy Eating Index are closely correlated with a reduced risk of diabetes, even with their different specific components ${ }^{(13)}$.

However, although the effects of a healthy diet on diabetes prevention are known, most studies are conducted in western populations in developed countries ${ }^{(14)}$. Therefore, the results of those studies may not be generalisable to populations with different dietary cultures in different regions, especially to certain Chinese populations who consume fewer whole grains and vegetables and more refined grains, red meat, unhealthy fats and fried foods ${ }^{(15)}$ such as in northern China.

Inner Mongolia is a frontier in northern China, located southeast of the Mongolian plateau, extending $2400 \mathrm{~km}$ east to west and $1700 \mathrm{~km}$ north to south. Inner Mongolia accounts for nearly one-eighth of the territory in China, which differs geographically and culturally from other regions in China. Because the typical gathering areas are multiethnic and dominated by Han Chinese or Mongolians, Inner Mongolian residents exhibit unique dietary habits. However, large-scale, representative nutritional diabetes-related epidemiological studies are lacking in Inner Mongolia, in particular, research that explored the relationship between dietary patterns and diabetes. Therefore, we conducted this study to understand the diabetes prevalence in Inner Mongolia and explore the dietary quality in association with the risk of diabetes.

\section{Methods}

\section{Study design}

This study was a surveillance survey of Chronic Disease and Nutrition Monitoring among Chinese Adults in Inner Mongolia in 2015. This was a cross-sectional, population-based survey that characterised the dietary and nondietary factors (i.e. general demographics, lifestyle, diabetes prevalence rate, dietary behavioural habits and daily food intake) among residents aged $\geq 18$ years in Inner Mongolia. The survey used a multistage-stratified cluster random sampling design covering eight monitoring sites including the urban, rural and pastoral areas in the eastern, central and western regions of Inner Mongolia.
The sample size estimation formula in this study according to the stratified random sampling was estimated based on the diabetes prevalence of the Health Service Survey in Inner Mongolia in $2012(P=0.0692)$. We considered a 0.015 permissible $\delta$ error value and a $10 \%$ non-response rate, and we estimated the sample size to be 1209 . In total, 1861 participants were included. According to the Health Service Survey in Inner Mongolia in 2012, the total number of participants surveyed was 21500 $(P=0.0692)$ and our study was $1861(P=0.085)$. The statistical power $(1-\beta)$ was $71 \%$.

The Ethical Committee of the National Institute for Nutrition and Food Safety, Chinese Center for Disease Control and Prevention approved the survey (201519-A). All participants provided written informed consent before the start of the investigation.

\section{Dietary data collection}

Dietary data were collected from a 24-h dietary recall and weighing method over three consecutive days. The dietary survey was in accordance with the Chinese Dietary Guidelines for chronic disease and nutrition surveillance in Chinese adults and aimed to determine residents' nutrient and food intake. All household members (aged $\geq 2$ years) were investigated. In the 24-h dietary recalls, participants recalled and described the types and quantities of food consumed over three consecutive days. Information on daily consumption of major seasonings, such as cooking oil, salt and monosodium glutamate, was collected using a weighing method. Condiments purchased and wasted were also recorded.

Drinking frequency, type (liquor with high alcohol content, liquor with low alcohol content, beer, yellow rice wine, rice wine, wine) and average drinking amount were measured. The average daily alcohol consumption was calculated according to the Manual of Chinese Chronic Disease and Nutrition Surveillance Survey ${ }^{(16)}$. Participants' height, weight and waist circumference were measured directly by trained investigators. Blood and urine samples were also collected. The laboratory director organised the sample quality control assessment at a field laboratory.

\section{Chinese Dietary Balance Index-16}

The Chinese Dietary Balance Index-16 (DBI-16) is a method of evaluating dietary structure and quality based on the Chinese Dietary Guidelines, consisting of eight components (value range): (i) cereals ( -12 to 12 ); (ii) vegetables ( -6 to 0 ) and fruits ( -6 to 0 ); (iii) milk ( -6 to 0 ) and soyabeans ( -6 to 0 ); (iv) animal foods ( -4 to 4 for meat, -4 to 0 for fish and -4 to 4 for eggs); (v) pure energy foods ( $0-6$ for oil, 0-6 for alcohol); (vi) condiments (0-6 for sugar; 0-6 for salt); (vii) dietary variety ( -12 to 0 ) and (viii) drinking water $(-12 \text { to } 0)^{(17)}$. The DBI-16 evaluation of intake quality for different foods is based on consumption patterns of individuals with different energy intakes. A score of 0 for each component indicates that the recommended intake has been met. Positive scores (0-12) indicate excess intake; negative scores ( -12 to 0$)$ indicate insufficient intake. The DBI-16 is further divided into twelve food subgroups used to calculate the dietary variety score ${ }^{(18)}$. 
The DBI-16 contains three dietary quality indicators: the higher-bound score (HBS), the lower-bound score (LBS) and diet quality distance (DQD) $)^{(17)}$. The HBS is calculated by adding all positive scores as an indicator of excessive food intake. The LBS is calculated by adding the absolute values of all negative scores as an indicator of insufficient food intake. The DQD is an indicator of unbalanced food intake, calculated by adding the absolute values of both the positive and negative scores. The possible ranges for the HBS, LBS and DQD scores are 0-44, 0-72 and 0-96, respectively. Each indicator is divided into five levels to reflect dietary quality: (i) 'excellent' (a score of 0); (ii) 'good' ( $<20 \%$ of the total score); (iii) 'low level' (20-40\% of the total score); (iv) 'moderate level' ( $40-60 \%$ of the total score) and (v) 'high level' ( $>60 \%$ of the total score $)^{(17,19)}$. The total score of each DBI-16 component is divided by the total LBS, HBS and DQD to assess their contribution rate and how much of each food subgroup affects dietary quality.

\section{Definition of diabetes}

The main outcome indicator was type 2 diabetes. Meeting one of the following conditions was considered to indicate diabetes. The first condition was self-reported diabetes, in which patients were diagnosed with diabetes, are currently receiving diabetes treatment and/or are taking hypoglycaemic drugs. The second condition was that as per the International Diabetes Federation ${ }^{(2)}$, diabetes was diagnosed if one or more of the following criteria were met: (1) fasting plasma glucose $\geq 7.0 \mathrm{mmol} / \mathrm{l}$; (2) glycated HbA1c $\geq 6.5 \%$; (3) 2-h plasma glucose $\geq 11.1 \mathrm{mmol} / 1$ following a $75 \mathrm{~g}$ oral glucose load and (4) random plasma glucose $\geq 11 \cdot 1 \mathrm{mmol} / \mathrm{l}$.

\section{Definition of other variables}

Age was categorised as $18-, 30-$ or $\geq 50$ years. Participant household registration location was categorised as urban or rural. Based on regional characteristics, ethnic groups were categorised as Han, Mongolian or other minorities (i.e. all minorities living in Inner Mongolia except Han and Mongolian). Educational level was categorised as low (primary school or lower), medium (junior high school) or high (senior high school and above). Marital status was categorised as married, unmarried or widowed/divorced.

Abdominal obesity was diagnosed as per the Working Group on Obesity of China ${ }^{(20)}$ : waist circumference $\geq 90 \mathrm{~cm}$ for men and waist circumference $\geq 85 \mathrm{~cm}$ for women.

Alcohol consumption was determined for the past year. Participants were categorised according to their average daily alcohol consumption: no drinking $(0 \mathrm{~g} / \mathrm{d})$, moderate drinking $(<25 \mathrm{~g} / \mathrm{d})$ or excessive drinking $(>25 \mathrm{~g} / \mathrm{d})^{(21)}$.

Smoking status was categorised as non-smoker (never having smoked previously), ex-smoker (previously smoked but quit) or current smoker (has smoked at least 1 cigarette/d for more than 1 year and currently smokes). In the present study, metabolic exercise equivalent was calculated by the International Physical Activity Questionnaire ${ }^{(22)}$ and physical activity was divided into low, medium and high levels.

\section{Statistical analysis}

Normally distributed continuous variables are presented as means and standard deviations; abnormally distributed continuous variables are presented as medians and interquartile ranges. Categorical variables are presented as numbers and percentages. Student's $t$ test and the Mann-Whitney-Wilcoxon $U$ test were used to assess two independent groups of continuous variables; the $\chi^{2}$ test was used to assess categorical variables.

Principal component analysis was used to derive dietary patterns based on twenty-nine food groups. The varimax rotation was used to extract factor loadings. Factors were selected based on their eigenvalues $(>1 \cdot 00)$. The number of dietary patterns was determined based on scree plots, reasonability of food combination and variance contribution rate. Dietary patterns were named by combining the food composition characteristics of the dietary pattern with the main food types included. Factor scores for each pattern were calculated by adding the coefficient of the factor loading and the standardised daily intake amounts of every food type related to each pattern. Scores for each dietary pattern were categorised into quartiles $\mathrm{Q}_{1}, \mathrm{Q}_{2}, \mathrm{Q}_{3}$ and $\mathrm{Q}_{4}$ in ascending order of factor scores. Higher factor scores indicated more compliance with the dietary pattern; lower scores indicated more deviation.

Dietary pattern quality was evaluated using generalised linear models, with LBS, HBS and DQD as the dependent variables and dietary patterns as the independent variable after adjusting for other confounders. Multivariate logistic regression models were used to examine the association between dietary patterns and diabetes. The 'Forward: LR' method was used to select independent variables. With $\alpha=0.05$ as the significance level, $P<0.05$ was considered statistically significant. All statistical analyses were conducted using IBM SPSS Statistics version 19.0 (IBM Corp.).

\section{Results}

\section{Participant characteristics}

We included 1861 participants: 973 women (52.3\%) and 888 men $(47.7 \%)$. The participants' mean age was 52.5 years. Of participants, 159 (8.5\%) had diabetes, and of these, eighty-six were men $(54.1 \%)$ and seventy-three were women $(45.9 \%)$. Of participants, $61.0 \%$ were over 50 years old, $58.4 \%$ were from rural areas and $18.2 \%$ were of ethnic minorities. Participants aged over 50 years had the highest diabetes prevalence at $11.6 \%$ $(P<0.05)$. Diabetes prevalence was higher in urban areas than in rural areas $(P<0.05)$, and thirty-nine participants $(12.6 \%)$ had low levels of physical activity. Diabetes prevalence was higher in participants with abdominal obesity than in those without abdominal obesity $(P<0.05)$ and in participants with dyslipidaemia than in those without dyslipidaemia $(P<0 \cdot 05)$. No other variables differed statistically $(P>0 \cdot 05$; Table 1$)$.

\section{Dietary patterns}

Principal component analysis identified four distinct dietary patterns. The most prominent dietary pattern (i.e. the pattern that explained the greatest amount of the total variance) was 
Table 1. Demographic and lifestyle characteristics of diabetic and nondiabetic participants ( $n$ 1861)

(Numbers and percentages)

\begin{tabular}{|c|c|c|c|c|c|}
\hline \multirow[b]{3}{*}{ Group } & \multicolumn{4}{|c|}{ Diabetes } & \multirow[b]{3}{*}{$P$} \\
\hline & \multicolumn{2}{|c|}{ No } & \multicolumn{2}{|c|}{ Yes } & \\
\hline & $n$ & $\%$ & $n$ & $\%$ & \\
\hline \multicolumn{6}{|l|}{ Sex } \\
\hline Men & 802 & $90 \cdot 3$ & 86 & $9 \cdot 7$ & \multirow[t]{2}{*}{0.093} \\
\hline Women & 900 & 92.5 & 73 & 7.5 & \\
\hline \multicolumn{6}{|l|}{ Ethnicity } \\
\hline Han & 1384 & $90 \cdot 9$ & 138 & $9 \cdot 1$ & \multirow[t]{3}{*}{$0 \cdot 230$} \\
\hline Mongolian & 254 & 93.7 & 17 & $6 \cdot 3$ & \\
\hline Other minority & 64 & $94 \cdot 1$ & 4 & $5 \cdot 9$ & \\
\hline \multicolumn{6}{|l|}{ Education level } \\
\hline Low & 771 & $90 \cdot 3$ & 83 & $9 \cdot 7$ & \multirow[t]{3}{*}{0.227} \\
\hline Medium & 539 & $92 \cdot 8$ & 42 & $7 \cdot 2$ & \\
\hline High & 392 & $92 \cdot 0$ & 34 & 8.0 & \\
\hline \multicolumn{6}{|l|}{ Marital status } \\
\hline Married & 1577 & 91.4 & 148 & $8 \cdot 6$ & \multirow[t]{3}{*}{0.788} \\
\hline Single & 45 & 93.8 & 3 & $6 \cdot 3$ & \\
\hline Widowed/divorced & 74 & $90 \cdot 2$ & 8 & $9 \cdot 8$ & \\
\hline \multicolumn{6}{|l|}{ Age group (years) } \\
\hline $18-$ & 86 & $97 \cdot 7$ & 2 & $2 \cdot 3$ & \multirow[t]{3}{*}{$<0.001$} \\
\hline $30-$ & 613 & $96 \cdot 1$ & 25 & 3.9 & \\
\hline$\geq 50$ & 1003 & 88.4 & 132 & 11.6 & \\
\hline \multicolumn{6}{|l|}{ Place of residence } \\
\hline Urban & 68 & 89.0 & 85 & $11 \cdot 0$ & \multirow[t]{2}{*}{0.001} \\
\hline Rural & 1013 & $93 \cdot 2$ & 74 & $6 \cdot 8$ & \\
\hline \multicolumn{6}{|l|}{ Income } \\
\hline Low & 805 & $91 \cdot 2$ & 78 & $8 \cdot 8$ & \multirow[t]{2}{*}{0.996} \\
\hline High & 763 & $91 \cdot 2$ & 74 & 8.8 & \\
\hline \multicolumn{6}{|l|}{ Drinking } \\
\hline Never & 1600 & 91.5 & 148 & 8.5 & \multirow[t]{3}{*}{0.893} \\
\hline Moderate & 45 & $90 \cdot 0$ & 5 & $10 \cdot 0$ & \\
\hline Excessive & 57 & $90 \cdot 5$ & 6 & 9.5 & \\
\hline \multicolumn{6}{|l|}{ Smoking status } \\
\hline Current smoker & 530 & $90 \cdot 3$ & 57 & $9 \cdot 7$ & \multirow[t]{3}{*}{0.080} \\
\hline Ex-smoker & 95 & $87 \cdot 2$ & 14 & $12 \cdot 8$ & \\
\hline Non-smoker & 1077 & 92.4 & 88 & $7 \cdot 6$ & \\
\hline Physical activity & & & & & \\
\hline Low level & 270 & $87 \cdot 4$ & 39 & $12 \cdot 6$ & 0.005 \\
\hline Medium level & 428 & 90.5 & 45 & 9.5 & \\
\hline High level & 1004 & 93.0 & 75 & $7 \cdot 0$ & \\
\hline Abdominal obesity & & & & & \\
\hline No & 1030 & $95 \cdot 8$ & 45 & $4 \cdot 2$ & $<0.001$ \\
\hline Yes & 637 & 84.8 & 114 & $15 \cdot 2$ & \\
\hline Dyslipidaemia & & & & & \\
\hline No & 1023 & 95.0 & 54 & 5.0 & $<0.001$ \\
\hline Yes & 679 & $86 \cdot 6$ & 105 & $13 \cdot 4$ & \\
\hline Total & 1702 & 91.5 & 159 & 8.5 & \\
\hline
\end{tabular}

'meat/dairy products' (eigenvalue: $2 \cdot 615 ; 9.017 \%$ of the total variance), characterised by milk tea and tea, fried wheat products, beef and mutton, milk and dairy products and had the highest observed positive loading factors. The second most prominent dietary pattern was 'traditional northern' (eigenvalue: $2.003 ; 6.908 \%$ of the total variance), representing a typical traditional diet: high intake of starchy roots and products, pork, pickled vegetables/dried vegetables, maize and desserts. The third most prominent dietary pattern (eigenvalue: 1.875; $6.456 \%$ of the total variance) was 'high cereal/tuber', characterised by intake of various vegetables, beans and bean products, wheat flour and products, fresh fruits, various aquatic products, eggs, vegetable oils, sugar, various beverages and nuts. The fourth (least prominent) dietary pattern was 'high-salt/alcohol' (eigenvalue: $1 \cdot 503 ; 5 \cdot 184 \%$ of the total variance), characterised by high intake of salt, animal oils, condiments and alcoholic beverages. Together, these four dietary patterns accounted for $27.754 \%$ of the variability in the original dietary variables. The Kaiser-Meyer-Olkin measure of sampling adequacy was 0.615 , and Bartlett's test of sphericity was significant $(P<0 \cdot 01)$, determining the suitability of the data set for the principal component analysis (shown as Table 2).

\section{Dietary pattern characteristics}

Table 3 shows the food group characteristics of the four dietary patterns. For 'meat/dairy products', the dietary factor scores of the different foods for cereals, meat, milk and dairy products, eggs, vegetables, fish and shrimp, tea, fried food and salt intake were statistically significant between $\mathrm{Q}_{1}$ and $\mathrm{Q}_{4}(P<0 \cdot 05)$. Among them, $\mathrm{Q}_{4}$ was higher than $\mathrm{Q}_{1}$ for cereals, meat, milk and dairy products, vegetables, tea, fried food and salt. Egg, fish and shrimp intakes were higher in $\mathrm{Q}_{1}$ than in $\mathrm{Q}_{4}$.

For 'traditional northern', the cereal, meat, vegetable and cooking oil intakes were higher in $\mathrm{Q}_{4}$ than in $\mathrm{Q}_{1}(P<0 \cdot 05)$. The milk and dairy product, fish and shrimp, tea and fried food intakes were lower in $\mathrm{Q}_{4}$ than in $\mathrm{Q}_{1}(P<0.05)$. The soyabean, egg, fruit and salt intakes were not statistically different $(P>0 \cdot 05)$.

For 'high cereal/tuber', the milk and dairy product, tea, fried food and salt intakes were lower in $\mathrm{Q}_{4}$ than in $\mathrm{Q}_{1}(P<0.05)$. Intake of other food was higher in $\mathrm{Q}_{4}$ than in $\mathrm{Q}_{1}(P<0.05)$.

For 'high-salt/alcohol', the dietary factor scores for the intake of foods other than fried foods were statistically significant between $\mathrm{Q}_{1}$ and $\mathrm{Q}_{4}(P<0 \cdot 05)$. Cereal, meat, soyabean, vegetable, fish and shrimp, cooking oil and salt intakes were higher in $\mathrm{Q}_{4}$ than in $\mathrm{Q}_{1}(P<0.05)$. Milk and dairy product, egg, fruit and tea intakes were lower in $\mathrm{Q}_{4}$ than in $\mathrm{Q}_{1}(P<0.05)$.

\section{Nutrient and energy intakes by dietary pattern}

Table 4 compares nutrient and energy intakes of the four dietary patterns.

For 'meat/dairy products', the carbohydrate, fat, protein, $\mathrm{Ca}, \mathrm{Mg}$, $\mathrm{K}, \mathrm{Na}$, dietary fibre and energy intakes from protein were higher in $\mathrm{Q}_{4}$ than in $\mathrm{Q}_{1}(P<0.05)$. Energy intake from carbohydrates was lower in $\mathrm{Q}_{4}$ than in $\mathrm{Q}_{1}(P<0 \cdot 05)$. For 'traditional northern', the carbohydrate, fat, protein, $\mathrm{Ca}, \mathrm{Mg}, \mathrm{K}$, dietary fibre and energy intakes from fat were higher in $\mathrm{Q}_{4}$ than in $\mathrm{Q}_{1}(P<0.05)$. For 'high cereal/tuber', energy intake from fat was lower in $Q_{4}$ than in $Q_{1}$ $(P<0.05)$ and the remaining nutrient and energy intakes were higher in $\mathrm{Q}_{4}$ than in $\mathrm{Q}_{1}(P<0 \cdot 05)$. For 'high-salt/alcohol', Ca intake and energy intake from carbohydrates and protein were lower $\mathrm{Q}_{4}$ than in $Q_{1}(P<0 \cdot 05)$, while other nutrient and energy intakes were higher in $\mathrm{Q}_{4}$ than in $\mathrm{Q}_{1}(P<0 \cdot 05)$.

\section{Quality evaluation of dietary patterns using Dietary Balance Index-16 scores}

Table 5 shows the generalised linear model of the dietary patterns and DBI-16. For 'meat/dairy products', the regression coefficients were all <0: $\beta_{\mathrm{LBS}}=-1.993(95 \% \mathrm{CI}-2.362,-1.625$; $P<0.001), \beta_{\mathrm{HBS}}=-0.206$ (95\% CI $\left.-0.381,-0.030 ; P=0.021\right)$ and $\beta_{\mathrm{DQD}}=-2 \cdot 199(95 \% \mathrm{CI}-2.598,-1.801 ; P<0.001)$. As the 
Table 2. Factor loadings of each dietary pattern among Inner Mongolian adults ( $n$ 1861) in northern China, 2015

\begin{tabular}{|c|c|c|c|c|}
\hline Food group & Meat/dairy product pattern & Traditional northern pattern & High cereal/tuber pattern & High-salt/alcohol pattern \\
\hline Milk tea and tea & 0.795 & _- & _- & - \\
\hline Fried wheat products & 0.727 & - & - & - \\
\hline Beef/mutton & 0.627 & - & - & - \\
\hline Dairy products & 0.416 & - & - & - \\
\hline Milk & 0.371 & - & - & - \\
\hline Potatoes & - & 0.780 & - & - \\
\hline Dried vegetables, pickles & - & 0.729 & - & - \\
\hline Pork & - & 0.651 & - & - \\
\hline Maize and other cereals & - & 0.198 & - & - \\
\hline Desserts, snacks, snack food & - & 0.120 & - & - \\
\hline Vegetables & - & - & 0.562 & - \\
\hline Soyabeans & - & - & 0.545 & - \\
\hline Wheat flour & - & - & 0.495 & - \\
\hline Fresh fruits & - & - & 0.458 & - \\
\hline Aquatic products & - & _- & 0.427 & _- \\
\hline Eggs & - & - & 0.425 & - \\
\hline Algae & - & - & 0.346 & - \\
\hline Vegetable oil & - & - & 0.254 & - \\
\hline Processed meat & - & - & 0.241 & - \\
\hline Starch and sugar & - & - & 0.236 & - \\
\hline Beverages & - & - & 0.114 & - \\
\hline Nuts & - & - & 0.105 & - \\
\hline Salt & - & - & - & 0.654 \\
\hline Animal oil & - & - & - & 0.607 \\
\hline Condiments & - & - & - & 0.528 \\
\hline Rice & - & - & - & 0.468 \\
\hline Alcohol & - & - & - & 0.318 \\
\hline Animal giblets & - & - & - & 0.180 \\
\hline Cake & - & - & - & -0.131 \\
\hline Variance & 9.017 & 6.908 & 6.465 & $5 \cdot 184$ \\
\hline
\end{tabular}

dietary pattern factor scores increased $\left(\mathrm{Q}_{1}-\mathrm{Q}_{4}\right)$, the dietary balance indexes of the LBS, HBS and DQD decreased and the diet was relatively balanced. For 'traditional northern', as the factor score increased $\left(\mathrm{Q}_{1}-\mathrm{Q}_{4}\right)$, the LBS decreased, the HBS and DQD increased, and the diet tended to be unbalanced. For 'high cereal/tuber', as the factor score increased $\left(\mathrm{Q}_{1}-\mathrm{Q}_{4}\right)$, the HBS increased, the LBS and DQD decreased, and the diet tended to be unbalanced. For 'high-salt/alcohol', the regression coefficients were all $>0: \quad \beta_{\mathrm{LBS}}=0.967 \quad(95 \%$ CI $0.570, \quad 1.364$; $P<0.001), \beta_{\mathrm{HBS}}=0.751$ (95\% CI 0.570, 0.933; $\left.P<0.001\right)$ and $\beta_{\mathrm{DQD}}=1.718(95 \%$ CI $1.293,2.143 ; P<0.001)$. As the dietary pattern factor scores increased $\left(Q_{1}-Q_{4}\right)$, the LBS, HBS and DQD increased and the diet was relatively unbalanced.

\section{Association between dietary patterns and diabetes}

Table 6 shows the relationship between dietary patterns and diabetes that was analysed using logistic regression model. After adjusting for sex, age, residence, educational level, marital status, ethnic group, smoking status, abdominal obesity and dyslipidaemia, the 'meat/dairy product' pattern was associated with diabetes risk. Compared with $\mathrm{Q}_{1}, \mathrm{Q}_{4}$ was associated with a lower risk of diabetes (OR 0.565; 95\% CI 0.338, 0.945; $P<0.05$ ). No other dietary patterns were related to diabetes prevalence.

\section{Discussion}

Diabetes prevalence is related to economic level and tends to increase with societal and economic development ${ }^{(23)}$. In this study, Inner Mongolia had an $8.5 \%$ diabetes prevalence, lower than that in high-income areas, such as Beijing ${ }^{(24)}$ and Shanghai ${ }^{(25)}$, but higher than that in Jiangxi ${ }^{(26)}$ and Guilin ${ }^{(27)}$. According to the age distribution of the 2010 population census in China, the estimated standardised diabetes prevalence was $6.0 \%$ in Inner Mongolia, which was lower than the national levels in $2013^{(28)}$ and $2019^{(2)}$. Therefore, diabetes is moderately prevalent in Inner Mongolia.

In Inner Mongolia, four dietary patterns were identified: 'meat/dairy products', 'traditional northern', 'high cereal/tuber' and 'high-salt/alcohol'. These dietary patterns accounted for $27.754 \%$ of the variability in the original dietary variables. And in other studies, the explicable variability for diet by dietary pattern ranged from 18.6 to $31.7 \%^{(29-33)}$. Our result was consistent with other studies and was even higher than some studies.

The relationship between dietary patterns and diabetes in participants showed that the 'meat/dairy product' pattern was negatively correlated with diabetes risk. This dietary pattern included dairy products, milk tea and tea, beef and mutton and fried wheat products. Evidence from another study showed that a higher intake of dairy products (e.g. milk, yogurt, cheese and whey protein) was beneficial for glucose regulation and reduced the risk of type 2 diabetes mellitus ${ }^{(34)}$. Fan et al. $^{(35)}$ showed that consuming $200 \mathrm{~g} / \mathrm{d}$ of milk reduced the risk of diabetes by $9 \%$. Dairy products have abundant nutrient elements such as protein, minerals and fat ${ }^{(36)}$. In our study, $13.4 \%$ of the energy consumed by the participants following the 'meat/ dairy product' pattern came from protein, which exceeds the recommended nutrient intake for Chinese residents ${ }^{(37)}$. A study 
Table 3. Food groups according to the lowest $\left(Q_{1}\right)$ and highest $\left(Q_{4}\right)$ quartile for each dietary pattern among Inner Mongolian adults $(n 1861)$ in northern China, 2015 (Medians and interquartile ranges (IQR))

\begin{tabular}{|c|c|c|c|c|c|c|c|c|c|c|c|c|c|c|c|c|c|c|c|c|}
\hline & \multicolumn{4}{|c|}{ Meat/dairy product pattern } & \multirow[b]{3}{*}{$P$} & \multicolumn{4}{|c|}{ Traditional northern pattern } & \multirow[b]{3}{*}{$P$} & \multicolumn{4}{|c|}{ High cereal/tuber pattern } & \multirow[b]{3}{*}{$P$} & \multicolumn{4}{|c|}{ High-salt/alcohol pattern } & \multirow[b]{3}{*}{$P$} \\
\hline & \multicolumn{2}{|r|}{$Q_{1}$} & \multicolumn{2}{|r|}{$\mathrm{Q}_{4}$} & & \multicolumn{2}{|r|}{$\mathrm{Q}_{1}$} & \multicolumn{2}{|r|}{$\mathrm{Q}_{4}$} & & \multicolumn{2}{|r|}{$\mathrm{Q}_{1}$} & \multicolumn{2}{|r|}{$\mathrm{Q}_{4}$} & & \multicolumn{2}{|r|}{$Q_{1}$} & \multicolumn{2}{|r|}{$\mathrm{Q}_{4}$} & \\
\hline & Median & IQR & Median & IQR & & Median & IQR & Median & IQR & & Median & IQR & Median & IQR & & Median & IQR & Median & IQR & \\
\hline Cereal $(\mathrm{g} / \mathrm{d})$ & 201.25 & $153 \cdot 75,273.05$ & 272.29 & $201 \cdot 52,395 \cdot 75$ & $<0.001$ & 184.00 & $137.05,228.98$ & 391.33 & $289.50,520 \cdot 00$ & $<0.001$ & $194 \cdot 38$ & $141 \cdot 82,272 \cdot 94$ & 339.17 & $250 \cdot 83,469.58$ & $<0.001$ & $230 \cdot 14$ & $174 \cdot 46,295 \cdot 88$ & 273.61 & $200 \cdot 00,404.79$ & $<0.001$ \\
\hline Meat $(\mathrm{g} / \mathrm{d})$ & 31.67 & $0.00,63.67$ & 116.67 & $66.67,188.78$ & $<0.001$ & 33.33 & $0.00,73.33$ & 110.67 & $50.00,189.45$ & $<0.001$ & 45.00 & $0.00,101.67$ & 83.00 & $33.33,150.00$ & $<0.001$ & 33.33 & $0.00,73.33$ & 73.33 & $30.00,144.73$ & $<0.001$ \\
\hline $\begin{array}{l}\text { Milk and dairy } \\
\text { products (g/d) }\end{array}$ & 0.00 & $0.00,0.00$ & 66.67 & $0.00,116.67$ & $<0.001$ & 0.00 & $0.00,137.22$ & 0.00 & $0.00,0.00$ & $<0.001$ & 0.00 & $0.00,100.00$ & 0.00 & $0.00,7.00$ & $<0.001$ & 83.33 & $0.00,166 \cdot 67$ & 0.00 & $0.00,0.00$ & $<0.001$ \\
\hline Soyabeans (g/d) & 0.00 & $0.00,18 \cdot 33$ & 0.00 & $0.00,33.33$ & 0.858 & 0.00 & $0.00,33.33$ & 0.00 & $0.00,34.50$ & 0.591 & 0.00 & $0.00,0.00$ & 33.33 & $0.00,83.33$ & $<0.001$ & 0.00 & $0.00,16 \cdot 67$ & 0.00 & $0.00,33 \cdot 33$ & 0.007 \\
\hline Eggs (g/d) & 0.00 & $0.00,33.33$ & 0.00 & $0.00,16.67$ & $<0.001$ & 0.00 & $0.00,33.33$ & 0.00 & $0.00,25 \cdot 96$ & 0.440 & 0.00 & $0.00,0.00$ & 19.17 & $0.00,50.00$ & $<0.001$ & 0.00 & $0.00,33.33$ & 0.00 & $0.00,18.50$ & $<0.001$ \\
\hline Vegetables $(g / d)$ & 60.00 & $27.50,110 \cdot 00$ & 116.67 & $60 \cdot 00,176 \cdot 33$ & $<0.001$ & 76.67 & $34.17,139.25$ & 96.67 & $33 \cdot 33,203 \cdot 33$ & 0.002 & 30.00 & $0.00,66.67$ & $162 \cdot 61$ & $104 \cdot 17,250.00$ & $<0.001$ & 83.33 & $33 \cdot 33,143 \cdot 33$ & 93.89 & $46 \cdot 25,159 \cdot 01$ & 0.018 \\
\hline Fruit (g/d) & 0.00 & $0.00,40.53$ & 0.00 & $0.00,65.87$ & 0.082 & 0.00 & $0.00,43.41$ & 0.00 & $0.00,6.90$ & 0.065 & 0.00 & $0.00,0.00$ & 65.87 & $0.00,153.13$ & $<0.001$ & 0.00 & $0.00,60.83$ & 0.00 & $0.00,40.13$ & 0.039 \\
\hline Fish and shrimps (g/d) & 0.00 & $0.00,0.00$ & 0.00 & $0.00,0.00$ & 0.036 & 0.00 & $0.00,0.00$ & 0.00 & $0.00,0.00$ & $<0.001$ & 0.00 & $0.00,0.00$ & 0.00 & $0.00,10.00$ & $<0.001$ & 0.00 & $0.00,0.00$ & 0.00 & $0.00,0.00$ & $<0.001$ \\
\hline Tea $(\mathrm{g} / \mathrm{d})$ & 0.00 & $0.00,0.00$ & 0.00 & $0.00,400.00$ & $<0.001$ & 0.00 & $0.00,0.00$ & 0.00 & $0.00,0.00$ & $<0.001$ & 0.00 & $0.00,0.00$ & 0.00 & $0.00,0.00$ & $<0.001$ & 0.00 & $0.00,0.00$ & 0.00 & $0.00,0.00$ & $<0.001$ \\
\hline Fried food $(\mathrm{g} / \mathrm{d})$ & 0.00 & $0.00,0.00$ & 33.33 & $0.00,66 \cdot 67$ & $<0.001$ & 0.00 & $0 \cdot 00,23 \cdot 33$ & 0.00 & $0.00,0.00$ & $<0.001$ & 0.00 & $0.00,0.00$ & 0.00 & $0.00,0.00$ & $<0.001$ & 0.00 & $0.00,0.00$ & 0.00 & $0.00,0.00$ & 0.056 \\
\hline Cooking oil (g/d) & 25.67 & $14 \cdot 52,44 \cdot 28$ & 26.00 & $13 \cdot 50,44.59$ & 0.526 & 24.67 & $12 \cdot 67,40 \cdot 68$ & 37.67 & $19.57,59 \cdot 17$ & $<0.001$ & $20 \cdot 10$ & $9.00,38.33$ & 35.33 & $21 \cdot 88,52 \cdot 33$ & $<0.001$ & 20.22 & $9 \cdot 17,34.88$ & 41.67 & $23 \cdot 29,65 \cdot 36$ & $<0.001$ \\
\hline Salt (g/d) & 6.36 & $4.00,11 \cdot 19$ & 7.35 & $4.92,11.38$ & $<0.001$ & 7.00 & $4.50,13.00$ & 8.63 & $5 \cdot 17,13 \cdot 77$ & 0.068 & 7.33 & $4.83,13.38$ & 7.00 & $4.83,10.67$ & 0.021 & 5.33 & $3.67,7.33$ & 12.42 & $7.33,20.67$ & $<0.001$ \\
\hline
\end{tabular}


Table 4. Nutrient and energy intakes according to the lowest $\left(Q_{1}\right)$ and highest $\left(Q_{4}\right)$ quartile of each dietary pattern among Inner Mongolian adults $(n 1861)$ in northern China, 2015 (Medians and interquartile ranges (IQR))

\begin{tabular}{|c|c|c|c|c|c|c|c|c|c|c|c|c|c|c|c|c|c|c|c|c|}
\hline & \multicolumn{4}{|c|}{ Meat/dairy product pattern } & \multirow[b]{3}{*}{$P$} & \multicolumn{4}{|c|}{ Traditional northern pattern } & \multirow[b]{3}{*}{$P$} & \multicolumn{4}{|c|}{ High cereal/tuber pattern } & \multirow[b]{3}{*}{$P$} & \multicolumn{4}{|c|}{ High-salt/alcohol pattern } & \multirow[b]{3}{*}{$P$} \\
\hline & \multicolumn{2}{|r|}{$Q_{1}$} & \multicolumn{2}{|r|}{$\mathrm{Q}_{4}$} & & \multicolumn{2}{|r|}{$Q_{1}$} & \multicolumn{2}{|r|}{$\mathrm{Q}_{4}$} & & \multicolumn{2}{|r|}{$Q_{1}$} & \multicolumn{2}{|r|}{$\mathrm{Q}_{4}$} & & \multicolumn{2}{|r|}{$Q_{1}$} & \multicolumn{2}{|r|}{$\mathrm{Q}_{4}$} & \\
\hline & Median & IQR & Median & IQR & & Median & IQR & Median & IQR & & Median & IQR & Median & IQR & & Median & IQR & Median & IQR & \\
\hline Carbohydrate (g/d) & $168 \cdot 12$ & $132 \cdot 38,210 \cdot 87$ & 231.62 & $185 \cdot 77,307 \cdot 25$ & $<0.001$ & 169.86 & $135 \cdot 06,225 \cdot 18$ & 246.49 & $196.59,317.56$ & $<0.001$ & 167.50 & $128.05,208.85$ & 265.17 & $206 \cdot 61,349 \cdot 33$ & $<0.001$ & 192.89 & $159.70,241.51$ & 223.22 & $168 \cdot 92,288 \cdot 76$ & $<0.001$ \\
\hline Total fat $(\mathrm{g} / \mathrm{d})$ & 47.49 & $29 \cdot 32,70 \cdot 59$ & 67.41 & $50 \cdot 50,96 \cdot 50$ & $<0.001$ & 49.38 & $31 \cdot 43,67.44$ & 84.81 & $56.81,122.89$ & $<0.001$ & $46 \cdot 10$ & $24 \cdot 61,70 \cdot 87$ & 69.02 & $51.08,92.80$ & $<0.001$ & 47.52 & $29 \cdot 47,65 \cdot 36$ & 69.91 & $46.94,106.97$ & $<0.001$ \\
\hline Protein $(\mathrm{g} / \mathrm{d})$ & 32.25 & $24 \cdot 22,42 \cdot 39$ & 63.47 & $48.56,82.93$ & $<0.001$ & 37.54 & $27 \cdot 82,52.97$ & 56.18 & $43.76,81.56$ & $<0.001$ & 32.94 & $25 \cdot 41,47.64$ & 61.03 & $48.03,83.54$ & $<0.001$ & 39.91 & $30 \cdot 20,52 \cdot 35$ & 48.40 & $35.13,65.82$ & $<0.001$ \\
\hline $\begin{array}{l}\text { Energy intake from } \\
\text { carbohydrates }(\%)\end{array}$ & 53.77 & $43 \cdot 44,62 \cdot 29$ & 51.26 & $44.62,57.54$ & 0.007 & 51.85 & $43.64,59.05$ & 50.90 & $42 \cdot 29,58 \cdot 25$ & 0.153 & 53.21 & $45.08,62.73$ & $52 \cdot 33$ & $45 \cdot 22,59 \cdot 55$ & 0.103 & 56.21 & $49 \cdot 51,63.63$ & 48.52 & $40.11,56.97$ & $<0.001$ \\
\hline Energy intake from fat (\%) & 33.27 & $23 \cdot 99,42 \cdot 52$ & 33.25 & $26 \cdot 98,39 \cdot 31$ & 0.720 & 32.00 & $23 \cdot 20,39 \cdot 27$ & 36.23 & $27 \cdot 34,45 \cdot 96$ & $<0.001$ & 33.57 & $24 \cdot 31,42 \cdot 35$ & 30.80 & $23 \cdot 58,38 \cdot 55$ & 0.004 & $30 \cdot 23$ & $22 \cdot 79,37 \cdot 38$ & 34.58 & $24 \cdot 87,45 \cdot 61$ & $<0.001$ \\
\hline $\begin{array}{l}\text { Energy intake from } \\
\text { protein (\%) }\end{array}$ & $10 \cdot 17$ & $8.53,11.82$ & $13 \cdot 40$ & $11 \cdot 17,15 \cdot 76$ & $<0.001$ & 11.71 & $9.54,14.45$ & 11.16 & $9 \cdot 52,13.58$ & 0.122 & 10.95 & $9.21,13.08$ & 12.04 & $10 \cdot 43,14.45$ & $<0.001$ & 11.68 & $10 \cdot 48,13 \cdot 11$ & 10.46 & $8 \cdot 11,13 \cdot 23$ & $<0.001$ \\
\hline $\mathrm{Ca}(\mathrm{mg} / \mathrm{d})$ & 165.79 & $121 \cdot 09,222 \cdot 62$ & 349.45 & $256 \cdot 66,442 \cdot 59$ & $<0.001$ & 254.69 & $189.46,353.64$ & 286.74 & $199 \cdot 47,405 \cdot 85$ & $<0.001$ & 195.04 & $125 \cdot 33,271 \cdot 51$ & $344 \cdot 32$ & $269 \cdot 26,454 \cdot 59$ & $<0.001$ & 278.96 & $207.84,372.06$ & 239.14 & $163.31,346.69$ & $<0.001$ \\
\hline $\mathrm{Mg}(\mathrm{mg} / \mathrm{d})$ & 165.63 & $123 \cdot 83,218 \cdot 36$ & 234.74 & $189.55,303.48$ & $<0.001$ & $161 \cdot 17$ & $120 \cdot 73,216 \cdot 39$ & 264.09 & $216 \cdot 34,344.58$ & $<0.001$ & $168 \cdot 17$ & $109 \cdot 12,228.39$ & 270.89 & $206 \cdot 79,344.75$ & $<0.001$ & 184.24 & $132.96,234.50$ & 226.37 & $171 \cdot 11,301.64$ & $<0.001$ \\
\hline$K(g / d)$ & 0.80 & $0.53,1.05$ & 1.44 & $1 \cdot 14,1.86$ & $<0.001$ & 0.89 & $0.60,1.23$ & 1.68 & $1 \cdot 30,2 \cdot 17$ & $<0.001$ & 0.83 & $0.55,1.25$ & 1.61 & $1.27,2.06$ & $<0.001$ & 1.03 & $0.70,1.39$ & 1.21 & $0.87,1.78$ & $<0.001$ \\
\hline $\mathrm{Na}(\mathrm{g} / \mathrm{d})$ & 3.44 & $2 \cdot 50,5 \cdot 70$ & 4.24 & $3 \cdot 17,5.98$ & $<0.001$ & 3.95 & $2 \cdot 66,6.88$ & 4.37 & $2.94,6.54$ & 0.324 & 3.90 & $2.66,6.29$ & 3.84 & $2 \cdot 81,5.66$ & 0.802 & 2.97 & $2 \cdot 28,4 \cdot 11$ & 6.67 & $4 \cdot 35,9.92$ & $<0.001$ \\
\hline Fibre $(g / d)$ & $5 \cdot 13$ & $3 \cdot 53,7 \cdot 27$ & $7 \cdot 10$ & $5 \cdot 31,10.52$ & $<0.001$ & 5.07 & $3 \cdot 31,7 \cdot 08$ & 8.51 & $6 \cdot 12,11 \cdot 84$ & $<0.001$ & 4.48 & $2.83,6.57$ & $10 \cdot 13$ & $7 \cdot 63,13 \cdot 10$ & $<0.001$ & 5.89 & $3.96,8 \cdot 87$ & 6.72 & $4 \cdot 83,9 \cdot 43$ & $<0.001$ \\
\hline
\end{tabular}


Table 5. Generalised linear model ${ }^{\star}$ of dietary quality according to the indicators of the Chinese Dietary Balance Index-16 (DBI-16) for each dietary pattern among Inner Mongolian adults $(n 1861)$ in northern China, 2015

(Regression coefficients (Coef.) and $95 \%$ confidence intervals)

\begin{tabular}{|c|c|c|c|c|c|c|c|c|c|}
\hline & \multicolumn{3}{|c|}{ LBS } & \multicolumn{3}{|c|}{ HBS } & \multicolumn{3}{|c|}{ DQD } \\
\hline & Coef. & $95 \% \mathrm{Cl}$ & $P$ & Coef. & $95 \% \mathrm{Cl}$ & $P$ & Coef. & $95 \% \mathrm{Cl}$ & $P$ \\
\hline Meat $/$ da & -1.993 & $-2 \cdot 362,-1 \cdot 625$ & $<0.001$ & -0.206 & $-0.381,-0.030$ & 0.021 & $-2 \cdot 199$ & $-2.598,-1.801$ & $<0.001$ \\
\hline Traditional northern pattern & -0.690 & $-1.084,-0.295$ & 0.001 & 1.567 & $1.398,1.735$ & $<0.001$ & 0.877 & $0.451,1.308$ & $<0.001$ \\
\hline High cereal/tuber pattern & -4.882 & $-5 \cdot 255,-4.508$ & $<0.001$ & 0.739 & $0.540,0.937$ & $<0.001$ & $-4 \cdot 143$ & $-4.574,-3.712$ & $<0.001$ \\
\hline High-salt/alcohol pattern & 0.967 & $0.570,1.364$ & $<0.001$ & 0.751 & $0.570,0.933$ & $<0.001$ & 1.718 & $1 \cdot 293,2 \cdot 143$ & $<0.001$ \\
\hline
\end{tabular}

LBS, lower-bound score; HBS, higher-bound score; DQD, diet-quality distance.

${ }^{*}$ Model adjusted for sex, age, place of residence, educational level, marital status, ethnic group, salt intake control, smoking status, weight control, BMI, hypertension and dyslipidaemia.

Table 6. Association of dietary patterns with diabetes across quartiles $(Q)$ of dietary pattern scores among Inner Mongolian adults $(n 1861)$ in northern China, $2015 \dagger$

(Odds ratios and $95 \%$ confidence intervals)

\begin{tabular}{|c|c|c|c|c|c|c|c|c|c|c|}
\hline & \multirow{2}{*}{$\frac{Q_{1}}{O R}$} & \multicolumn{2}{|c|}{$Q_{2}$} & \multirow[b]{2}{*}{$P$} & \multicolumn{2}{|c|}{$\mathrm{Q}_{3}$} & \multirow[b]{2}{*}{$P$} & \multicolumn{2}{|c|}{$\mathrm{Q}_{4}$} & \multirow[b]{2}{*}{$P$} \\
\hline & & OR & $95 \% \mathrm{Cl}$ & & OR & $95 \% \mathrm{Cl}$ & & OR & $95 \% \mathrm{Cl}$ & \\
\hline \multicolumn{11}{|c|}{ Meat/dairy product pattern } \\
\hline Model 1 & 1.000 & 0.741 & $0.468,1.174$ & 0.202 & 0.998 & $0.649,1.534$ & 0.991 & 0.673 & $0.420,1.078$ & 0.099 \\
\hline Model 2 & 1.000 & 0.590 & $0.360,0.968$ & 0.037 & 0.691 & $0.432,1.107$ & 0.124 & 0.565 & $0.338,0.945$ & $0.030^{*}$ \\
\hline \multicolumn{11}{|c|}{ Traditional northern pattern } \\
\hline Model 1 & 1.000 & 0.929 & $0.597,1.445$ & 0.743 & 0.833 & $0.530,1.309$ & 0.427 & 0.738 & $0.463,1.175$ & 0.201 \\
\hline Model 2 & 1.000 & 0.950 & $0.590,1.531$ & 0.833 & 0.752 & $0.461,1.227$ & 0.254 & 0.811 & $0.489,1.345$ & 0.417 \\
\hline \multicolumn{11}{|c|}{ High cereal/tuber pattern } \\
\hline Model 1 & 1.000 & 1.223 & $0.766,1.953$ & 0.400 & 1.005 & $0.617,1.635$ & 0.985 & 1.381 & $0.874,2 \cdot 183$ & 0.167 \\
\hline Model 2 & 1.000 & 1.208 & $0.726,2.088$ & 0.467 & 0.786 & $0.461,1.338$ & 0.375 & $1 \cdot 172$ & $0.696,1.971$ & 0.551 \\
\hline \multicolumn{11}{|c|}{ High-salt/alcohol pattern } \\
\hline Model 1 & 1.000 & $1 \cdot 106$ & $0.716,1.709$ & 0.650 & 0.903 & $0.573,1.421$ & 0.658 & 0.677 & $0.417,1.099$ & 0.115 \\
\hline Model 2 & 1.000 & 1.156 & $0.728,1.834$ & 0.540 & 0.956 & $0.592,1.546$ & 0.856 & 0.688 & $0.407,1.164$ & 0.164 \\
\hline
\end{tabular}

* $P<0.05$

† Model 1: crude model. Model 2: adjusted for sex, age, place of residence, educational level, marital status, ethnic group, smoking status, abdominal obesity and dyslipidaemia.

showed that higher protein intake from plants and certain animal foods helped reduce the risk of diabetes ${ }^{(34)}$. A meta-analysis revealed that high-quality dietary fat was associated with a lower diabetes risk ${ }^{(38)}$. Dairy fat contributed to the protective relationship between dairy product intake and type 2 diabetes, and highfat dairy products were negatively correlated with diabetes incidence $^{(39)}$. The median $\mathrm{Ca}$ intake of participants adhering to the 'meat/dairy product' pattern was $349.5 \mathrm{mg} / \mathrm{d}$, which was lower than the recommended nutrient intake, but higher than that of the other three dietary patterns in Inner Mongolia. A cross-sectional analysis showed that $\mathrm{Ca}$ was associated with a lower prevalence of the metabolic syndrome and impaired fasting glycaemia and type 2 diabetes ${ }^{(40)}$.

For 'meat/dairy products', rice was not a main food composition. Individuals adhering to this dietary pattern were more likely to eat less or no rice. The rice intake may have been decreased, which partly explains the beneficial effect of this dietary pattern on reducing diabetes risk. Some studies have shown that consuming more white rice was positively correlated with the risk of type 2 diabetes mellitus, especially in Asian countries ${ }^{(41,42)}$. This may be related to the milling process in which white rice loses many of the antidiabetic fibres, vitamins and minerals in the grain ${ }^{(43,44)}$. White rice also has a higher glycaemic index ${ }^{(45)}$, which may be why white rice carries a higher diabetes risk. Additionally, in our study, vegetable and fruit intakes were lower among residents adhering to the 'meat/dairy product' pattern than that suggested by recommended nutrient intake. Research has suggested that increasing vegetable and fruit consumption helps with weight loss, which might indirectly reduce the type 2 diabetes mellitus incidence $^{(46)}$; however, some meta-analyses found no relationship between fruit and/or vegetable consumption and diabetes risk $^{(47,48)}$. Huang et al. ${ }^{(49)}$ showed that fruits with a moderate or high glycaemic index were positively associated with the occurrence of gestational diabetes mellitus. Therefore, consuming more fruits and vegetables may not help prevent diabetes. In our research, the 'meat/dairy product' pattern was negatively correlated with diabetes, possibly because fewer vegetables and fruits were consumed. However, this study was cross-sectional, and the relationship between vegetables and fruits and diabetes risk requires a longterm population intervention experiment.

Except for the 'meat/dairy product' pattern, our results showed no association between the other three dietary patterns and diabetes. Among them, the 'traditional northern' pattern was characterised by high potato, pork, pickled/dried vegetable, maize and dessert intake, which is similar to the typical traditional diet in northern China ${ }^{(50)}$. Characteristics of the 'high cereal/tuber' pattern were more vegetables, fresh fruits and aquatic products; thus, this dietary pattern is similar to the Dietary Approaches to Stop Hypertension diet but involved more cereals and meat, and the carbohydrate and $\mathrm{Na}$ intakes were higher than recommended. 
Excessive alcohol and salt intake characterised the 'high-salt/alcohol' pattern, in which oil and salt intake was higher than recommended, and the vegetable, egg and dairy product intakes were lower than recommended. Therefore, we found no evidence that these dietary patterns help reduce the risk of diabetes.

The relationship between dietary patterns and diabetes risk varies among populations with different dietary cultures in different regions and racial/ethnic groups ${ }^{(51,52)}$. Inner Mongolia across the eastern, central and western regions of north China has a multiethnic culture, thus formed a unique dietary culture. Although some a priori methods can assess a population's compliance with a specific dietary pattern, such as the Mediterranean and Dietary Approaches to Stop Hypertension and so on, but these high-quality diets included special foods and components such as olive oil and seafood. For geographical and climatic reasons, these types of foods are relatively lacking in Inner Mongolia. Thus, our study used the DBI-16 to assess the dietary quality in participants. The results showed that participants more adhered to the 'meat/dairy product' pattern, the dietary quality was relatively balanced. In our research, the relatively balanced dietary pattern was associated with a lower risk of diabetes, which was consistent with other study results ${ }^{(53,54)}$.

Our study had some limitations. Using a cross-sectional study prevents drawing conclusions about causality between dietary patterns and diabetes prevalence. More cohort and randomised controlled trials are needed. Although we simultaneously adjusted for major sociodemographic characteristics and lifestyle factors, residual unknown or unmeasured confounding factors may have been present.

\section{Conclusions}

In summary, Inner Mongolia exhibited a moderate diabetes prevalence and four major dietary patterns were identified. Among them, the dietary quality of the 'meat/dairy product' pattern as evaluated via the DBI-16 was relatively balanced and was correlated with a decreased risk of diabetes prevalence. However, more prospective studies are needed to determine the relationship between dietary quality and diabetes in Inner Mongolia.

\section{Acknowledgements}

The authors wish to thank the study participants for their contribution to the research, as well as current and past investigators and staff.

This study was supported by National Natural Science Foundation of China (no. 81960603) (Study on the Mechanism of NAMPT Regulated by Hyperglycemia and CpnT Promoting Macrophage Death in Diabetes with Pulmonary Tuberculosis Infection) and Natural Science Foundation of Inner Mongolia (no. 2019MS08112) (Epidemiological Study on Obesity and Metabolic Abnormalities in Adults in Inner Mongolia). The funding sources had no role in the design, analysis or writing of this article.

The research idea was conceived by X. W., data processing and analysis were conducted by S. T., H. L., R. S., J. W., M. X., Y. Q. and W. W. and the manuscript was written by S. T. and H. L. All authors read and approved the final manuscript. The authors declare that there are no conflicts of interest.

\section{Availability of data and materials}

The data that support the findings of this study are available from the National Institute for Chinese Center for Disease Control and Prevention but restrictions apply to the availability of these data, which were used under license for the current study, and so are not publicly available.

\section{References}

1. NCD Risk Factor Collaboration (2016) Worldwide trends in diabetes since 1980: a pooled analysis of 751 population-based studies with 4.4 million participants. Lancet 387, 1513-1530.

2. International Diabetes Federation (2019) IDF Diabetes Atlas Ninth Edition 2019. Brussels: IDF.

3. Ma D, Sakai H, Wakabayashi C, et al. (2017) The prevalence and risk factor control associated with noncommunicable diseases in China, Japan, and Korea. J Epidemiol 27, 568-573.

4. Tripathy JP (2018) Burden and risk factors of diabetes and hyperglycemia in India: findings from the Global Burden of Disease Study 2016. Diabet Metab Syndr Obes 11, 381-387.

5. Herrera MCA, Subhan FB \& Chan CB (2017) Dietary patterns and cardiovascular disease risk in people with type 2 diabetes. Curr Obes Rep 6, 405-413.

6. Schwingshackl L, Hoffmann G, Lampousi A-M, et al. (2017) Food groups and risk of type 2 diabetes mellitus: a systematic review and meta-analysis of prospective studies. Eur $J$ Epidemiol 32, 363-375.

7. Ley SH, Hamdy O, Mohan V, et al. (2014) Prevention and management of type 2 diabetes: dietary components and nutritional strategies. Lancet 383, 1999-2007.

8. Vitale M, Masulli M, Calabrese I, et al. (2018) Impact of a Mediterranean dietary pattern and its components on cardiovascular risk factors, glucose control, and body weight in people with type 2 diabetes: a real-life study. Nutrients 10, 1067.

9. Shirani F, Salehi-Abargouei A \& Azadbakht L (2013) Effects of Dietary Approaches to Stop Hypertension (DASH) diet on some risk for developing type 2 diabetes: a systematic review and meta-analysis on controlled clinical trials. Nutrition 29, 939-947.

10. Chiuve SE, Fung TT, Rimm EB, et al. (2012) Alternative dietary indices both strongly predict risk of chronic disease. J Nutr $\mathbf{1 4 2}$, 1009-1018

11. InterAct Consortium (2014) Adherence to predefined dietary patterns and incident type 2 diabetes in European populations: EPIC-InterAct Study. Diabetologia 57, 321-333.

12. Whitton C, Rebello SA, Lee J, et al. (2018) A healthy Asian a posteriori dietary pattern correlates with a priori dietary patterns and is associated with cardiovascular disease risk factors in a multiethnic Asian population. J Nutr 148, 616-623.

13. Jannasch F, Kröger J \& Schulze MB (2017) Dietary patterns and type 2 diabetes: a systematic literature review and metaanalysis of prospective studies. J Nutr 147, 1174-1182.

14. Yu D, Zheng W, Cai H, et al. (2018) Long-term diet quality and risk of type 2 diabetes among urban Chinese adults. Diabetes Care 41, 723-730.

15. Wang J, Lin X, Bloomgarden ZT, et al. (2020) The Jiangnan diet, a healthy diet pattern for Chinese. J Diabetes 12, 365-371.

16. Wang S, Lay S, Yu H, et al. (2016) Dietary guidelines for Chinese residents (2016): comments and comparisons. J Zhejiang Univ Sci B 17, 649-656.

17. He Y, Fang Y \& Xia J (2018) Update of the Chinese diet balance index: DBI-16. Acta Nutrimenta Sinica 40, 526-530. 
18. He D, Qiao Y, Xiong S, et al. (2020) Association between dietary quality and prediabetes based on the diet balance index. Sci Rep 10, 3190.

19. Zang J, Yu H, Zhu Z, et al. (2017) Does the dietary pattern of Shanghai residents change across seasons and area of residence: assessing dietary quality using the Chinese Diet Balance Index (DBI). Nutrients 9, 251.

20. Beifan Zhou, Coorperative Meta-Analysis Group Of China Obesity Task Force (2002) Predictive values of body mass index and waist circumference to risk factors of related diseases in Chinese adult population. Chin J Epidemiol 1, 5-10.

21. Chinese Nutrition Society (2016) The Chinese Dietary Guidelines (2016). Beijing: People's Medical Publishing House.

22. The IPAQ Group (2015) Guidelines for Data Processing and Analysis of the International Physical Activity Questionnaire. http://www.ipaq.ki.se

23. Shaw JE, Sicree RA \& Zimmet PZ (2010) Global estimates of the prevalence of diabetes for 2010 and 2030. Diabetes Res Clin Pract 87, 4-14.

24. Wang Y, Han X, Li Z, et al. (2015) Change trend in diabetes prevalence and related factors among 60-79 years old residents in Chaoyang district of Beijing, 2008-2014. Chin J Public Health 31, 907-911.

25. Fan E, Ma C \& Han W (2015) Investigation and analysis on risk factors of diabetes and pre-diabetes among residents in suburban community of Pudong District of Shanghai. Occup Health 31, 2703-2706.

26. Liu J, Zhu L, Li A, et al. (2011) Survey on epidemic situation and influencing factors of diabetes mellitus among urban and rural residents in Jiangxi Province. Pract Prev Med 18, 1637-1638.

27. Guo Z, Shi W, Ma M, et al. (2015) A study on the prevalence and economic burden of disease of hypertension and diabetes in Guilin City. Chin J Dis Control Prev 19, 887-889+893.

28. Wang L, Gao P, Zhang M, et al. (2017) Prevalence and ethnic pattern of diabetes and prediabetes in China in 2013. J Am Med Soc Assoc 317, 2515-2523.

29. Link LB, Canchola AJ, Bernstein L, et al. (2013) Dietary patterns and breast cancer risk in the California Teachers Study cohort. Am J Clin Nutr 98, 1524-1532.

30. Schwedhelm C, Iqbal K, Knüppel S, et al. (2018) Contribution to the understanding of how principal component analysisderived dietary patterns emerge from habitual data on food consumption. Am J Clin Nutr 107, 227-235.

31. Kolahdooz F, Ibiebele TI, Pols JC, et al. (2009) Dietary patterns and ovarian cancer risk. Am J Clin Nutr 89, 297-304.

32. Esmaillzadeh A, Kimiagar M, Mehrabi Y, et al. (2007) Dietary patterns and markers of systemic inflammation among Iranian women. J Nutr 137, 992-998.

33. Williams DE, Prevost AT, Whichelow MJ, et al. (2000) A crosssectional study of dietary patterns with glucose intolerance and other features of the metabolic syndrome. Br J Nutr $\mathbf{8 3}$, 257-266.

34. Comerford KB \& Pasin G (2016) Emerging evidence for the importance of dietary protein source on glucoregulatory markers and type 2 diabetes: different effects of dairy, meat, fish, egg, and plant protein foods. Nutrients $\mathbf{8}, 446$.

35. Fan M, Li Y, Wang C, et al. (2019) Dietary protein consumption and the risk of type 2 diabetes: a dose-response meta-analysis of prospective studies. Nutrients 11, 2783.
36. Tunick MH \& Hekken DLV (2015) Dairy products and health: recent insights. J Agric Food Chem 63, 9381-9388.

37. Chinese Nutrition Society (2014) Reference Intake of Dietary Nutrients for Chinese Residents (2013). Beijing: Standards Press of China.

38. Merino J, Guasch-Ferré M, Ellervik C, et al. (2019) Quality of dietary fat and genetic risk of type 2 diabetes: individual participant data meta-analysis. BMJ 366, 14292.

39. Ericson U, Hellstrand S, Brunkwall L, et al. (2015) Food sources of fat may clarify the inconsistent role of dietary fat intake for incidence of type 2 diabetes. Am J Clin Nutr 101, 1065-1080.

40. Fumeron F, Lamri A, Khalil CA, et al. (2011) Dairy consumption and the incidence of hyperglycemia and the metabolic syndrome: results from a French prospective study, Data from the Epidemiological Study on the Insulin Resistance Syndrome (DESIR). Diabetes Care 34, 813-817.

41. Nanri A, Mizoue T, Noda M, et al. (2010) Rice intake and type 2 diabetes in Japanese men and women: the Japan Public Health Center-based Prospective Study. Am J Clin Nutr 92, 1468-1477.

42. Villegas R, Liu S, Gao Y, et al. (2007) Prospective study of dietary carbohydrates, glycemic index, glycemic load, and incidence of type 2 diabetes mellitus in middle-aged Chinese women. Arch Intern Med 167, 2310-2316.

43. Slavin JL, Martini MC, Jacobs DR Jr, et al. (1999) Plausible mechanisms for the protectiveness of whole grains. Am J Clin Nutr 70, 459s-463s.

44. Schulze MB, Schulz M, Heidemann C, et al. (2007) Fiber and magnesium intake and incidence of type 2 diabetes: a prospective study and meta-analysis. Arch Intern Med 167, 956-965.

45. Foster-Powell K, Holt SHA \& Brand-Miller JC (2002) International table of glycemic index and glycemic load values: 2002. Am J Clin Nutr 76, 5-56.

46. Rolls BJ, Ello-Martin JA \& Tohill BC (2004) What can intervention studies tell us about the relationship between fruit and vegetable consumption and weight management? Nutr Rev 62, 1-17.

47. Hamer M \& Chida Y (2007) Intake of fruit, vegetables, and antioxidants and risk of type 2 diabetes: systematic review and meta-analysis. J Hypertens 25, 2361-2369.

48. Carter P, Gray LJ, Troughton J, et al. (2010) Fruit and vegetable intake and incidence of type 2 diabetes mellitus: systematic review and meta-analysis. BMJ 341, c4229.

49. Huang W, Lu Y, Xu M, et al. (2017) Excessive fruit consumption during the second trimester is associated with increased likelihood of gestational diabetes mellitus: a prospective study. Sci Rep 7, 43620.

50. Wang D, He Y, Li Y, et al. (2011) Dietary patterns and hypertension among Chinese adults: a nationally representative cross-sectional study. BMC Public Health 11, 925.

51. Ma RCW \& Chan JCN (2013) Type 2 diabetes in East Asians: similarities and differences with populations in Europe and the United States. Ann N Y Acad Sci 1281, 64-91.

52. Weng J, Zhou Z, Guo L, et al. (2018) Incidence of type 1 diabetes in China, 2010-13: population based study. BMJ 360, j5295.

53. Cheung LTF, Chan RSM, Ko GTC, et al. (2018) Diet quality is inversely associated with obesity in Chinese adults with type 2 diabetes. Nutr J 17, 63.

54. McNaughton SA, Dunstan DW, Ball K, et al. (2009) Dietary quality is associated with diabetes and cardio-metabolic risk factors. J Nutr 139, 734-742. 